\title{
sciendo
}

RESEARCH PAPERS FACULTY OF MATERIALS

SCIENCE AND TECHNOLOGY IN TRNAVA

SLOVAK UNIVERSITY OF TECHNOLOGY

IN BRATISLAVA

2018, Volume 26, Number 43

DOI 10.2478/rput-2018-0027

\section{TRACE ELEMENTS ANALYSIS BY PIXE SPECTROSCOPY}

\author{
Matúš BEŇO, Jozef DOBROVODSKÝ, Dušan VAŇA, \\ Stanislav MINÁRIK, Róbert RIEDLMAJER \\ SLOVAK UNIVERSITY OF TECHNOLOGY IN BRATISLAVA, \\ FACULTY OF MATERIALS SCIENCE AND TECHNOLOGY IN TRNAVA, \\ ADVANCED TECHNOLOGIES RESEARCH INSTITUTE, \\ UliCA JÁNA BOTTU 2781/25, 91724 TRNAVA, SLOVAK REPUBLIC \\ e-mail: matus.beno@stuba.sk, jozef.dobrovodsky@stuba.sk,dusan.vana@stuba.sk, \\ stanislav.minarik@stuba.sk, robert.riedlmajer@stuba.sk \\ Received: 04.06.2018, Accepted: 13.12.2018, Published: 29.01.2019
}

\begin{abstract}
The trace element analysis system is presented using Proton Induced X-ray Emission (PIXE) analysis at a new Ion Beam Centre in Trnava. Standard PIXE system dedicated to the measurement of thick solid samples was extended by a new application for trace element analysis in aerosol samples. The sample holder was modified with respect to the dimensions of the aerosol filters, and a new sample holder and a Faraday cup (FC) were made. The first results of the PIXE aerosol analysis are presented in this paper. Furthermore, the geometric efficiency of the detection system was verified using $55 \mathrm{Fe}$ radioactive source emitting monoenergetic Mn X-ray lines. The measured data were compared with the Monte Carlo simulations regarding/disregarding the $X$-ray attenuation.
\end{abstract}

\section{Key words}

PIXE, trace elements, ion beam, aerosol, geometric efficiency

\section{INTRODUCTION}

A new 6 MV Tandetron tandem ion accelerator at the Advanced Technologies Research Institute (ATRI) of the Slovak University of Technology, Faculty of Materials Science and Technology (STU MTF) in Trnava, was commissioned in December 2015. The system provides a proton beam from $500 \mathrm{keV}$ to $12 \mathrm{MeV}$ and is equipped with the Ion Beam Analysis (IBA) end-station. The available non-destructive analytical methods include Particle Induced X-ray Emission (PIXE), Rutherford Backscattering Spectrometry (RBS), Elastic Recoil Detection Analysis (ERDA) and Nuclear Reaction Analysis (NRA) (1,2).

PIXE is fast, highly sensitive (in ppm limits), precise, accurate and is better for analysis of both matrix and trace elements in a thick sample as compared to other ion beam analytical techniques $(3,4)$. The PIXE analysis can be performed for a large range of elements $(\mathrm{Na} \leq \mathrm{Z} \leq \mathrm{U})$ of the periodic table. The advantage of the PIXE technique (it offers qualitative and quantitative elemental analysis) is its non-destructive nature and a simultaneous multi- 
elemental character of analysis, having a wide range of applications in biology, medicine, atmospheric aerosol analysis, earth science, archeology, etc. (5).

Sensitivity of the PIXE analysis predetermines it for the use in monitoring the elemental composition of airborne aerosols and monitoring its pollution. Many trace elements are emitted into the atmosphere via both human activity and natural background (6). Natural sources include soil particles from ground surfaces, gases from volcanoes, forest fires, biological respiration, whereas anthropogenic sources are actually man-made sources including gases from industries, automobiles, coal thermal plants, refuse burning, etc. (7). A PIXE system for the analysis of trace element pollutants in air aerosols is presented.

\section{PIXE MEASUREMENT OF AEROSOL SAMPLES}

The basic PIXE experimental setup was extended and optimized for the aerosol samples measurement. The aerosols were collected at the thin polycarbonate membrane filter of $25 \mathrm{~mm}$ in diameter and $0.8 \mu \mathrm{m}$ pore size by the SKC standard 244-44MTX personal pump with an IOM sampler.

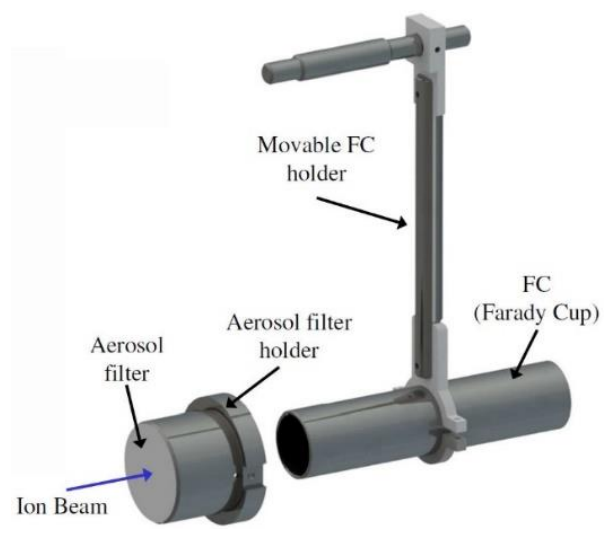

Fig. 1 PIXE aerosol setup

To minimize the background X-ray signal, the front site of the original sample holder was modified so that analyzing protons passing the aerosol filter continue freely to the new Faraday Cup (FC) situated at the backside of the IBA chamber. The beam current measurement by the new FC was tested. The PIXE setup was designed for normal incidence of the ion beam on the aerosol filter.

The obtaining PIXE spectra were evaluated by GUPIXWIN software. Before using the GUPIXWIN, many parameters that characterize the detection setting must be determined. Using the appropriate parameters, it si possible to analyze both elemental composition and concentration present in the target (8). 


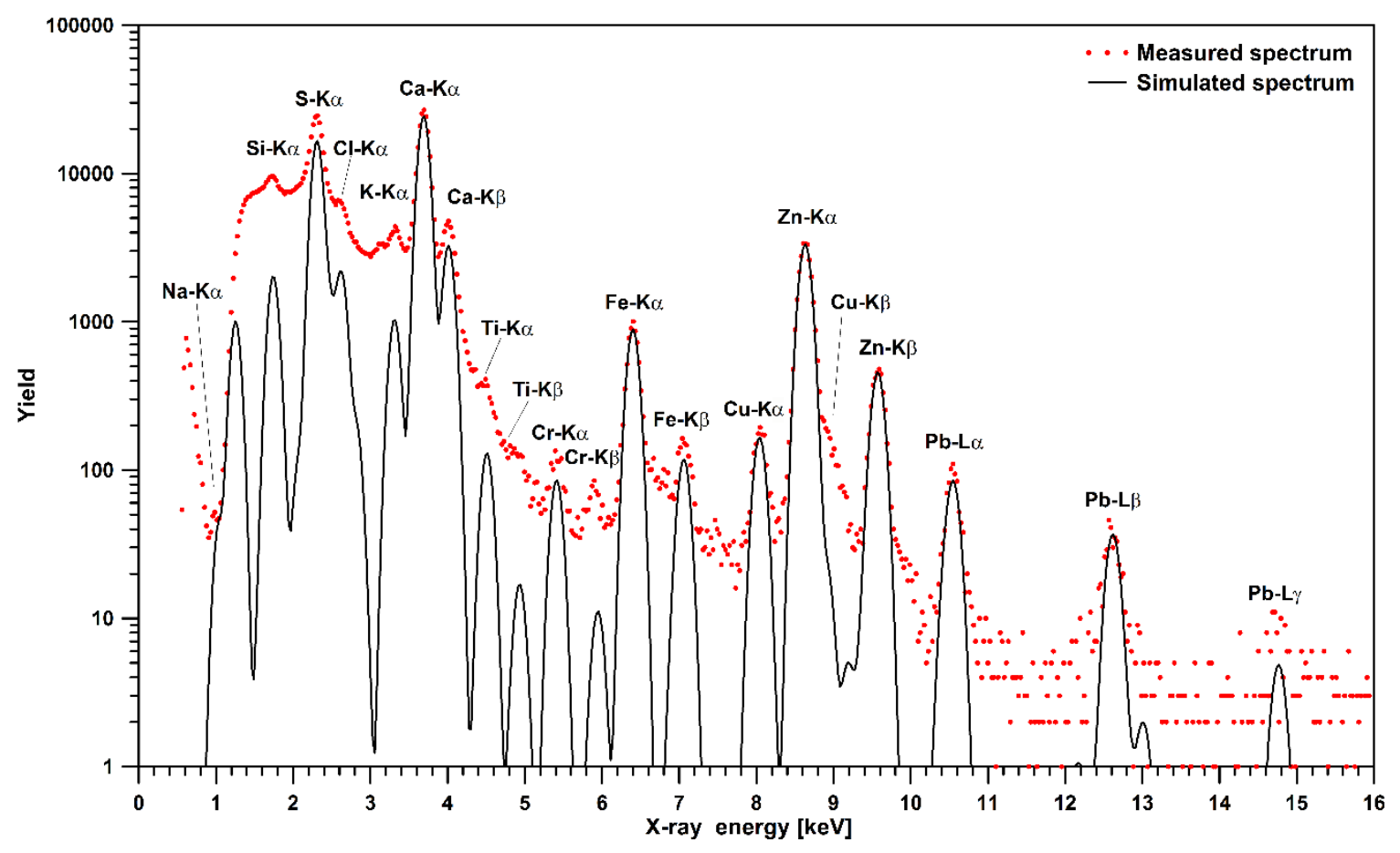

Fig. 2 PIXE logarithmic spectrum of aerosol sample

In our measurement of aerosol samples, the $2.5 \mathrm{MeV}$ energy incident proton beam, $2 \mathrm{nA}$ current and a dose of $30 \mu \mathrm{C}$ were used. The proton beam was collimated to a diameter of $5 \mathrm{~mm}$ spot. The X-rays were detected by a High Purity Germanium HPGe (GL0055) X-ray detector, with a $25 \mu \mathrm{m}$ thick Be window with no absorber foil, placed at $45^{\circ}$ to the beam axis. The energy resolution $150 \mathrm{eV}$ FWHM (Full Width at Half Maximum) at $5.9 \mathrm{keV}$, was acquired experimentally, using 55Fe radionuclide. The aerosol sample was clamped in a modified aerosols filter holder.

Figure 2 represents the qualitative analysis offering only the information regarding the type identification of trace elements composition of aerosol on the filer. The peak areas under individual detected X-ray lines are proportional to the corresponding element content. For quantitative evaluation of the measured spectra and in order to obtain elemental concentration, many input parameters such as detector efficiency (geometric efficiency and intrinsic efficiency), detector solid angle, stopping powers, attenuation coefficients etc. must be determined. The procedure to obtain the detector geometric efficiency is described in more detail below.

PIXE spectrum consists of the peaks which are approximately Gaussian, sitting on the top of a continuous background. The background-subtracted area of each peak is proportional to the concentration of the associated element, and a number of software packages is now available to perform the processing required to extract the areas and convert these to true concentrations.

For a sample consisting of a thin film (so that the proton energy loss and X-ray absorption can be neglected), the yield of the characteristic $X$-ray photons of energy $E_{x}$ from element $Z$ induced by the particles of energy $\mathrm{E}$ is given by

$$
\mathrm{N}=\mathrm{C}_{\mathrm{z}} \mathrm{Q} \Omega \varepsilon\left(E_{\mathrm{x}}\right) \mathrm{Y}(Z, E),
$$

where $\mathrm{N}$ is the total number of photons detected using a detector of solid angle $\Omega, \mathrm{Q}$ is the total beam charge, $\mathrm{C}_{\mathrm{Z}}$ is the concentration of the element and $\varepsilon\left(E_{\mathrm{x}}\right)$ is the energy dependent detector efficiency. $\mathrm{Y}(Z, E)$ is the yield of the interaction expressed as counts per unit of concentration per unit of charge per unit of solid angle. This, in turn, is derived from the ionization cross- 
section (the probability of creating a vacancy) and the fluorescence yield (the number of photons emitted in each X-ray line for each vacancy) (9).

\section{MONTE CARLO SIMULATION OF $X$-RAY DETECTOR GEOMETRIC EFFICIENCY}

Constant dimensions of both the source radius $R_{z}$ (flat disc) and detector active radius $R_{d}$ were used for our investigation. The source distance from the detector $d$ varied within the range from 5 to $250 \mathrm{~mm}$; $d_{\text {of }}$ being the distance between the detector crystal and the detector protection part. The next step of PIXE analysis of aerosol samples was quantitative verification of the obtained impurity concentrations using GUPIXWIN code. The $X$-ray detection geometric efficiency was verified using the monoenergetic radioactive source $55 \mathrm{Fe}$ emitting $\mathrm{Mn} \mathrm{X}$-ray lines. The distance between radioactive source and detector varied in range of 5 to $250 \mathrm{~mm}$ (full range). The measurements at each distance were taken for 600 seconds. The results of the measurement were compared with the data obtained from the Monte-Carlo simulation of the detection process. The section below explains the simulation algorithm and presents the calculated data processing.

Let $\vec{r}_{0}$ be parametric representation of the active area of X-ray emitter and $\vec{r}_{1}$ be parametric representation of the active area of X-ray detector (see fig.3). In the coordinate form:

$$
\begin{aligned}
& \vec{r}_{0}=\left[x_{0}(u, v), y_{0}(u, v), z_{0}(u, v)\right] \\
& \vec{r}_{1}=\left[x_{1}(\rho, w), y_{1}(\rho, w), z_{1}(\rho, w)\right]
\end{aligned}
$$

where parameters:

$$
\rho \in\left\langle\rho_{1}, \rho_{2}\right\rangle, \quad w \in\left\langle w_{1}, w_{2}\right\rangle, \quad u \in\left\langle u_{1}, u_{2}\right\rangle, \quad v \in\left\langle v_{1}, v_{2}\right\rangle .
$$

Direction of the $X$-ray photon propagation can be characterized by the unit vector:

$$
\vec{s}=[\cos (\varphi) \sin (\vartheta), \sin (\varphi) \sin (\vartheta), \cos (\vartheta)]
$$

Therefore, the photon position at time $t$ can be determined as:

$$
\vec{r}=\vec{r}_{0}+\vec{s} \tau
$$

where $\tau$ is (dimensionless) parameter.

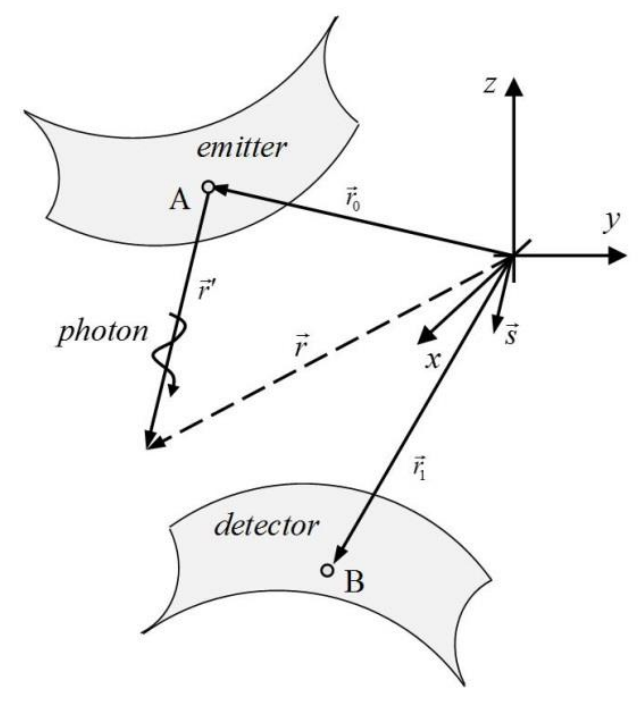

Fig. 3 A scheme illustrating an emitter-detector system and propagation of photon phenomena 
The photon hit the detector just when the top of vector $\vec{r}$ is located somewhere on the detector surfyce $\left(\vec{r}=\vec{r}_{1}\right)$. This means that in the case of photon interaction with the detector it applies:

In the case if:

$$
\begin{aligned}
& x_{0}(u, v)+\tau \cos (\varphi) \sin (\vartheta)=x_{1}(\rho, w) \\
& y_{0}(u, v)+\tau \sin (\varphi) \sin (\vartheta)=y_{1}(\rho, w) \\
& z_{0}(u, v)+\tau \cos (\vartheta) \quad=z_{1}(\rho, w) .
\end{aligned}
$$

$$
\vartheta \neq \frac{\pi}{2}
$$

the time $t$ when the photon impacts the detector can be found from the equation [8] as:

$$
\tau=\frac{z_{1}(\rho, w)-z_{0}(u, v)}{\cos (\vartheta)} .
$$

Considering the [9] in equations [6] and [7], we get:

$$
\begin{aligned}
& \left\{z_{1}(\rho, w)-z_{0}(u, v)\right\} \cos (\varphi) \operatorname{tg}(\vartheta)=x_{1}(\rho, w)-x_{0}(u, v) \\
& \left\{z_{1}(\rho, w)-z_{0}(u, v)\right\} \sin (\varphi) \operatorname{tg}(\vartheta)=y_{1}(\rho, w)-y_{0}(u, v) .
\end{aligned}
$$

Investigation of the solvability of the system [10] and [11] is the key to decision whether the photon hits the detector or not. If for the given (randomly selected) values $u, v, \varphi$ and $\vartheta$ this system has a solution $\rho$ a $w$ belonging to the defined intervals, the photon hits the detector.

The emitter-detector design in case if the active areas of X-ray emitter and detector have a coaxial cylindrical shape is illustrated in the Fig.4, where $R_{z}=3 \mathrm{~mm}$ is radius of the X-ray emitter, $R_{d}=4 \mathrm{~mm}$ is radius of the X-ray detector, $a$ is distance of the detector from emitter, $\vec{r}_{0}$ (A) is the position of the X-ray photon generation, $\vec{r}^{\prime}$ is the direction of the X-ray photon propagation and $\vec{r}(\mathrm{~B})$ is the (possible) position of the $\mathrm{X}$-ray photon absorption. In such a case:

$$
\begin{aligned}
& \vec{r}_{0}=[u \cos (v), u \sin (v), 0] \quad \ldots v \in\langle 0,2 \pi\rangle \ldots u \in\left\langle 0, R_{z}\right\rangle \\
& \vec{r}_{1}=[\rho \cos (w), \rho \sin (w),-a] \ldots \rho \in\langle 0,2 \pi\rangle \ldots w \in\left\langle 0, R_{d}\right\rangle
\end{aligned}
$$

and system [10] and [11] takes the following form:

$$
\begin{aligned}
& u \cos (v)-a \cos (\varphi) \operatorname{tg}(\vartheta)=\rho \cos (w) \\
& u \sin (v)-a \sin (\varphi) \operatorname{tg}(\vartheta)=\rho \sin (w) .
\end{aligned}
$$

The condition of the photon's impact on the detector must be obtained from the system [14] and [15] and can be written as follows:

$$
\{u \cos (v)-a \cos (\varphi) \operatorname{tg}(\vartheta)\}^{2}+\{u \sin (v)-a \sin (\varphi) \operatorname{tg}(\vartheta)\}^{2} \leq R_{d}^{2} .
$$




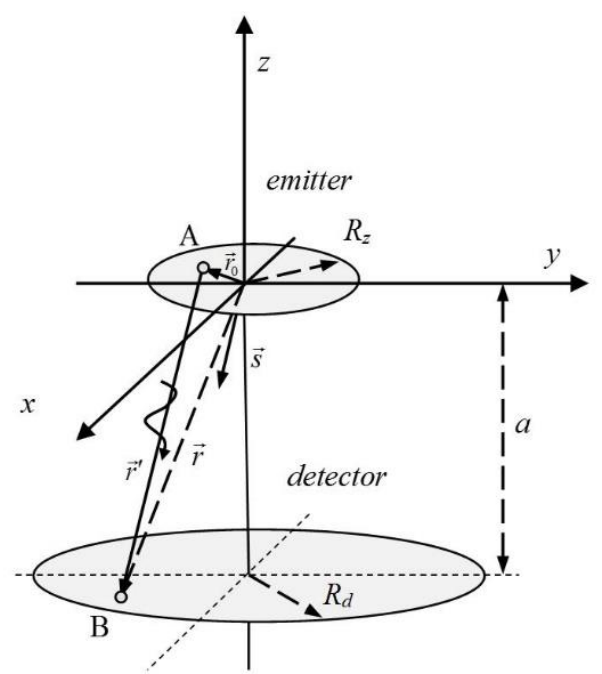

Fig. 4 Scheme emitter-detector system with cylindrical design

The following simple simulation algorithm based on the equations [14] and [15] was used for the data processing:

Random generation of position of the photon emission from the surface of the emitter (coordinates of vector $\vec{r}_{0}$ ):

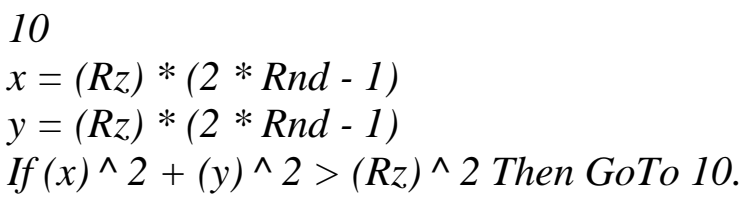

Random generation of the propagation direction of the photon unit vector (coordianteso of unit vector $s$ ):

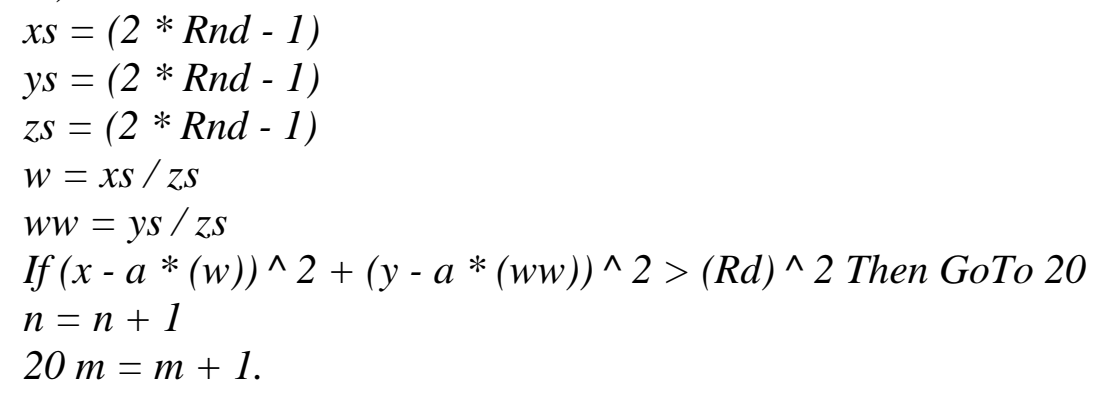

The total number of photons incident on the detector resulted from the simulation. Ratio between the calculated amount of photons and total number of photons emitted from the X-ray source indicates the total geometric efficiency of detector. The efficiency multiplied by intrinsic efficiency of detector should determine the measured data. The data obtained by simulation was modified using statistical methods and compared with experimental results. The results calculated for different values of $d_{\text {of }}$ were evaluated on the basis of statistical variations. We found that some attenuation of the X-rays between the source and the detector must be considered for the best fit of the experimental data (see Fig.5). We found that the attenuation can be described by standard formula of Lambert-Beer type:

$$
I=I_{0} e^{-\mu d} .
$$

The linear attenuation coefficient $\mu$ and detector offset $d_{\text {of }}$ were determined by simple optimalization procedure. The root mean square errors calculated from the measured data for various chosen $d_{o f}$ are listed in Table 1. 


\begin{tabular}{|c|c|}
\hline \multicolumn{2}{|c|}{$\begin{array}{c}\text { Table 1: Root mean square of calculated } \\
\text { errors for various } d_{o f}\end{array}$} \\
\hline$d_{\text {of }}[\mathrm{mm}]$ & $\begin{array}{c}\text { Root Mean Square } \\
\text { Error }[\text { Counts] }\end{array}$ \\
\hline 0 & 1271.85 \\
\hline 1 & 1086.84 \\
\hline 2 & 815.95 \\
\hline 3 & 608.81 \\
\hline 4 & 435.42 \\
\hline 5 & 368.69 \\
\hline $\mathbf{6}$ & $\mathbf{3 1 0 . 3 1}$ \\
\hline 7 & 326.69 \\
\hline 8 & 365.75 \\
\hline 9 & 437.59 \\
\hline 10 & 491.99 \\
\hline
\end{tabular}

As can be seen from this results, the best fit can be achieved for $d_{o f}=6 \mathrm{~mm}$. The linear attenuation coefficient corresponding to the value of $d_{\text {of }}$ was calculated as $\mu=0.0082233 \mathrm{~mm}^{-}$ ${ }^{1}$ and detector intrinsic efficiency was evaluated as $\varepsilon=0.06877$. Data fitting obtained for mentioned optimized simulation parameters is shown in graps in Fig.5.

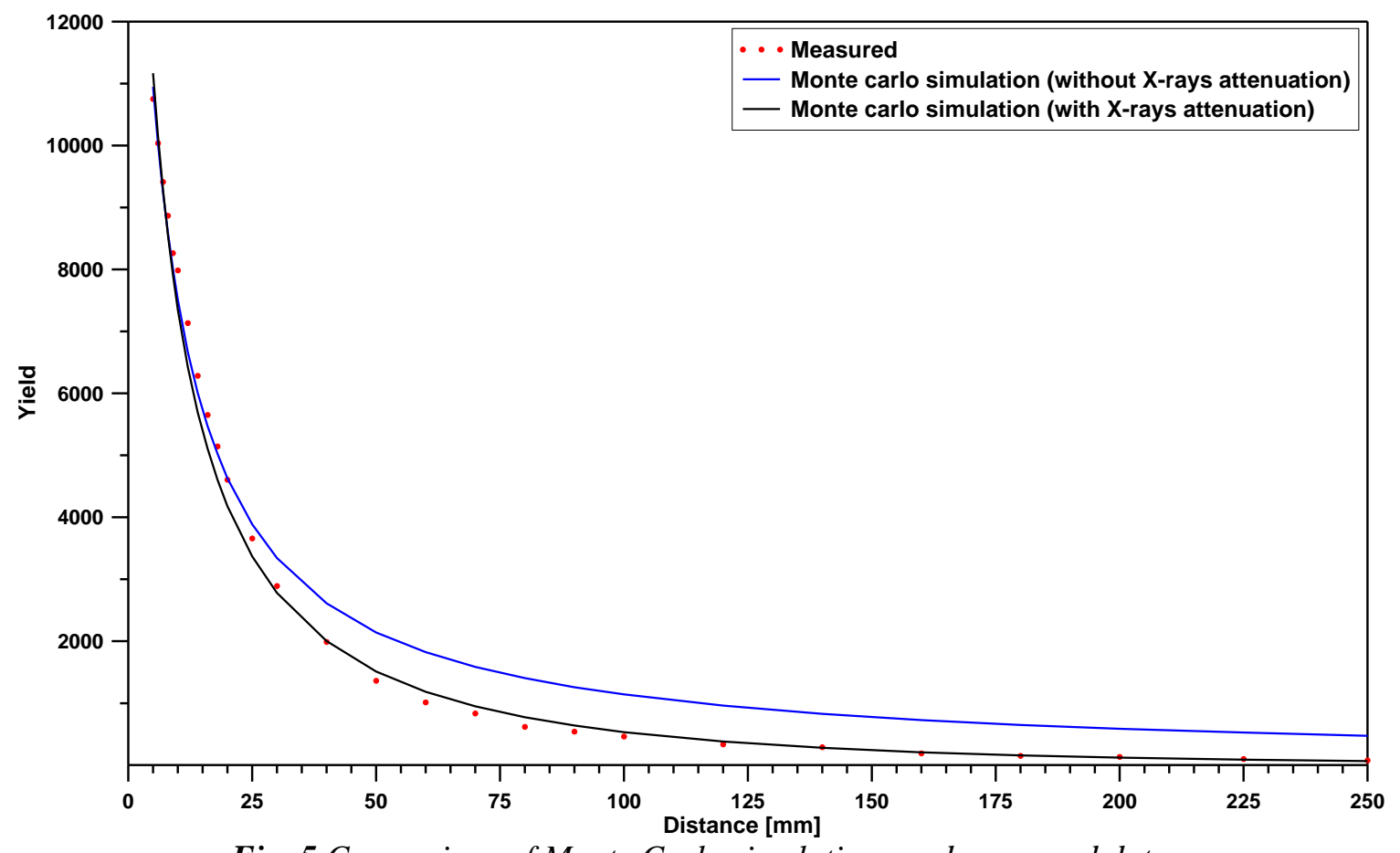

Fig. 5 Comparison of Monte Carlo simulations and measured data

\section{CONCLUSION}

The PIXE system at MTF ATRI in Trnava was extended by a new modified aerosol holder and the Faraday Cup situated behind the holder. Using the new setup and the GUPIXWIN software, the aerosol filters were qualitatively analyzed.

In conclusion, we reported the problem of Particle Induced X-ray Emission (PIXE) spectroscopy. Some partial aspects of the quantitative description of the primary PIXE yield were discussed. In this research, the Monte Carlo algorithm was applied to describe the PIXE detection process in some specific conditions, and to find the geometric efficiency of X-rays detector. As we can see from the results presented above, the experimental data correspond to 
the simulation results just in the case if the X-rays attenuation is considered. The agreement achieved between theoretical results and experimental data was quite satisfying for the cylindrical emitter-detector system.

Procedures of the simulation method suggested above will have to be modified in the case of other type of detection system, and the modification will be the subject of the future research. However, we think that principles of the method presented in our paper will remain the same. Generally, a question to ask is: what are the optimal Monte Carlo simulation parameters to the best fit of the measured data for a given geometry of the X-rays detector and emitter? Further systematic analysis of a great number of experimental data is necessary to find the answer to this question.

\section{Acknowledgement}

This research output was supported by the Research and Development Operational Programme under the project "University Scientific Park Campus MTF STU - CAMBO" ITMS: 26220220179, and Scientific Grant Agency of the Ministry of Education of Slovak Republic and the Slovak Academy of Sciences No. VEGA-1/0219/16.

\section{References:}

1. GOTTDANG, A., MOUS, D.J.W., HAITSMA, R.G. 2002. Nucl. Instrum. Meth. Phys. Res. B 190, 177.

2. NOGA, P., DOBROVOdSKÝ, J., VAŇA, D., BEŇO, M., ZÁVACKÁ, A., MUŠKA, M., HALGAŠ, R., MINÁRIK, S., RIEDLMAJER, R. 2017. A new ion-beam laboratory for materials research at the Slovak University of Technology. In: Nucl. Instr. Meth. Phys. Res. B, Volume 409, pp. 264-267, DOI: 10.1016/j.nimb.2017.04.051 [IF: 1.389].

3. JOHANSSON S. A. E., JOHANSSON T. B. 1976. Nuclear Instrument and Methods 137, 473.

4. ENE, A., POPESCU, I. V., STIHI, C., GHEBOIANU, A., PANTELICA, A., PETRE, C. 2009. Rom. Jour. Phy 55, 806.

5. HOANSSON, S. A. E., CAMPBELL, J. L., MJALMQVIST, K. G. Particle Induced X-ray Emission Spectroscopy (New York: John Willey \& Sons) pp. 147-412.

6. OGUlEI, D., HOPKE, P. K., ZHOU, L., PAATERO, P., PARK, S. S., ONDOV, J. M. 2005. Atmospheric Environment, 39, pp. 3751-3762.

7. OSWAL, M., KAUR, R., KUMAR, A., SINGH, K. P. 2012. International Journal of PIXE, Vol. 22, Nos. 3\&4, pp. 271-285.

8. ZEB, J., ALI, S., AHAD, A., AHMAD, I., AKBAR, J. 2017. Standardization of Proton Induced $X$-Ray Emission for Analysis of Trace Elements. Cornell University Library, arXiv:1703.04532 2017.

9. LINDON, J., TRANTER, G., KOPPENAAL, D. (eds.) 2010. Encyclopedia of Spectroscopy and Spectrometry. 2nd edition. Oxford: Elsevier; Vol 3., pp. 2247-2252. ISBN 9780123744173. 